\title{
HYBRID- AND NANOCOMPOSITES BASED ON THERMOELASTOPLASTICS FILLED IN BASALT SCALES AND NANO METALS AND ITS OXIDES
}

\author{
Vera Myasoedova, Elena Zakharova \& Ilja Vasiljev
}
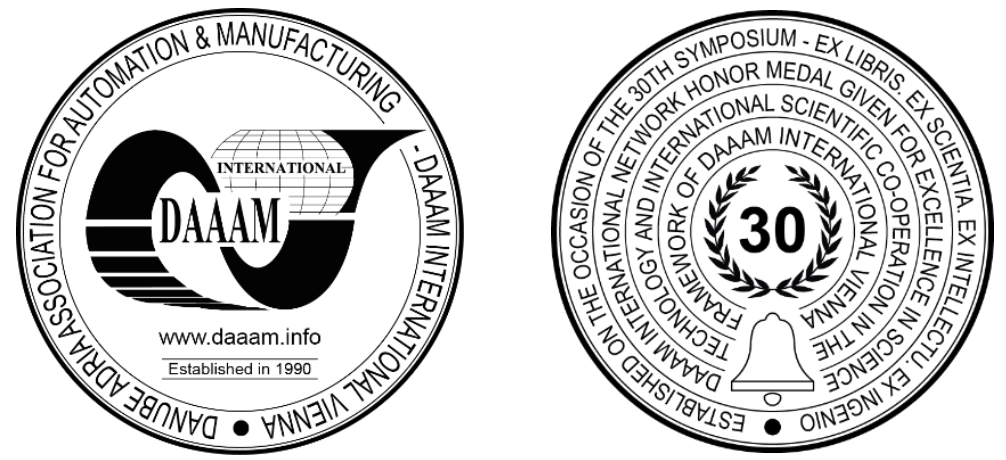

This Publication has to be referred as: Myasoedova, V[era]; Zakharova, E[lena] \& Vasiljev, I[lja] (2021). Hybrid- and Nanocomposites Based on Thermoelastoplastics Filled in Basalt Scales and nano Metals and Its Oxides, Proceedings of the 32nd DAAAM International Symposium, pp.0177-0184, B. Katalinic (Ed.), Published by DAAAM International, ISBN 978-3-902734-33-4, ISSN 1726-9679, Vienna, Austria

DOI: $10.2507 / 32$ nd.daaam.proceedings.027

\begin{abstract}
This article aims to develop innovations in the area of obtaining the hybrid- and polymer nanocomposites and investigation of its structural, thermodynamics' and physico-mechanical properties. As promising and extraordinary materials, nano metals, its oxides and hydroxides have been emerging in many areas of technology. The excellent properties of nanofiller makes this polymer nanocomposite material an ideal for manufacture of the new goods obtained by extrusion and improve some properties of polymer matrices. The success of the process depends on the obtainment of a homogeneous dispersion of nanoparticles without exfoliation. Investigation focuses on the melting process by extrusion and properties of innovation hybrid- and nanocomposite and material. Results open the possibility to evaluate the influence of different filler and polymer matrix chemical nature, sizes and content on increasing the thermostability and elasticity of the new hybrid- and nanocomposites. It is aiming the moulding mentioned above hybrid- and nanocomposites in form of plates, films, irrigation hoses for industrial application.
\end{abstract}

Keywords: Nano metals; polymer; nanocomposites; elastic properties.

\section{Introduction}

The use of hybrid composites and nanocomposites based on filled in micron- and nanosized particles of synthetic and natural thermoplastic and thermoelastic polymer materials as an alternative to replace traditional materials is of great of industrial, economic and environmental interest [1], [2],[3],[4]. A hybrid- and nanocomposite material can be defined as a multiphase composite material, containing at least two components, with an interface or interphase separating them. Choice of components should apply predetermined chemical, physical, mechanical (such as flexibility and elasticity or stiffness, or thermal stability of hybrid- and nanocomposites. The components are divided into two main phases: the matrix phase, which is a continuous phase and corresponds to the component with the highest content in the composition (> $50 \mathrm{wt} \%$ ), and the dispersed phase, also called reinforcement or filler that is a discontinuous phase of the component in less quantity. 
The use of hybrid composites and nanocomposites based on filled in micron- and nanosized particles of synthetic and natural thermoplastic and thermoelastic polymer materials as an alternative to replace traditional materials is of great industrial, economic and environmental interest. A hybrid- and nanocomposite material can be defined as a multiphase composite material, containing at least two components, with an interface or interphase separating them. The methods for nano metals synthesis described by [5], [6]. Historically, the first reference of the study of nanocomposites was in the 1950s, and studies of polyamide nanocomposites appeared in the 1970s. However, it was only at the end of the 1980s that there was a deepening of the subject with research carried out by Toyota's Central Research and Development Laboratories for the development of nanocomposites composed of polymers and nanoceramics, causing research on nanocomposites to evolve for an industrial scale [7].

The nanocomposites are classified according to the filler's nanometric dimensions (0 to $100 \mathrm{~nm})$ [8]. Nanometric fillers (nanofillers) have specific characteristics and induce different behaviours in the matrix when compared to micro and macro fillers, so, these materials can be considered as promising alternatives for use as materials that overcome the limitations of regular fillers. Some mechanical and physical properties which cannot be achieved in polymer composites made with regular fillers can be improved with the use of nanofillers. Nanofillers have a high surface/volume ratio, which allows the addition of smaller amounts $(1-5 \% \mathrm{v} / \mathrm{v})$, when compared to micrometric fillers $(15-40 \% \mathrm{v} / \mathrm{v})$, obtaining similar results of mechanical behaviour. Also, the surface/volume ratio of the nanofillers can induce better interaction between filler and matrix, which can be improved by the functionalization of the nanofiller Choice of components should apply predetermined chemical, physical, mechanical (such as flexibility and elasticity or stiffness, or thermal stability of hybridand nanocomposites. The components are divided into two main phases: the matrix phase, which is a continuous phase and corresponds to the component with the highest content in the composition (> $50 \mathrm{wt} \%$ ), and the dispersed phase, also called reinforcement or filler that is a discontinuous phase of the component in less quantity [2], [3]. The concern in its use is based on the fact that the variation between the components and their contents in the composition allows to obtain a range of materials with unique properties, many times different from the properties of their precursor materials. If the combination of these materials creates synergy between them, the properties of the hybrid- and nanocomposites may even outgrow the properties of the precursors. The composite materials can be classified based in: a) Matrix constituent: ceramic-matrix composites (CMC), metal-matrix composites and organic-matrix composites (OMC), that can be divided in carbon-matrix composites (carbon-carbon composites) and polymer-matrix composites (PMC); b) Type of reinforcement: scales, wickers, fibbers and particles; c) Size of fillers: macro-composites, micro- composites and nanocomposites. Hybrid- and nanocomposites are multiphase materials, as well as composite materials, however, at least one of its phases must be present on a nanometric scale, which may be one, two, or three nanometric dimensions [6]. The nanometric phase must also present one of its dimensions in the order of 1 and $100 \mathrm{~nm}$, obtaining a nanocomposite with excellent properties. However, nanofillers can have some difficulties related to their high surface/volume ratio, which can generate clusters (or agglomerates) or, mainly in high contents, and it can be formed during the processing of the nanocomposite or even in the production of the nanoparticle. For this reason, it is necessary to study the best methodology for dispersing the nanofillers (particles or fibres) to create a more homogeneous microstructure, otherwise, these aggregations or agglomerations could lead the nanocomposite to a heterogeneous dispersion, producing an unbalanced material and with loss of the interfacial area, presenting lower than expected properties. There are several ways to minimize agglomerates of powder particles of fillers is to choose a suitable methodology to develop a homogeneous dispersion of the fillers in the matrix. There are several ways to produce hybrid- and nanocomposites from existing (In situ polymerization: solution mixing (blending); melt mixing (melt blending): the micro- or nanofiller dispersion occurs directly in the polymer. In the last method process going in mixers, moulding injectors, or extruders. The mixing is carried out between the molten or rubbery state polymer and the micro- and nanofillers. Other techniques: there is also the possibility of choosing the best nanocomposite production technique for your application and adjust it in the way to produce new techniques, such as layering, hand lay-up, spray lay-up, sol-gel process, electrospinning, centrifugal casting, additive manufacturing, among others [7].

As founded by us the methodology chosen to extrusion process for the hybrid- and nanocomposites depends on several factors: the choice of the polymer matrix (individual synthetic or natural polymer, or their blends, containing thermoplastics (including, for example, polyolefins: polyethylene, polypropylene) and thermo elasto plastics (styrenebutadiene-styrene (SBS), styrene-ethylene-butadiene-styrene (SEBS), copolymer ethylene-octene (POE Solumer) and the micro- and nanofillers (rubber powder, active dyes, pigments dyestuff, as published by one of the authors of this paper $[8,10]$. Also the novel technical solutions and results are in the development of temperatures and concentrations regimes of extrusion process, the geometry and of the final product in form of films, strands, the application area - for containers and flexible eyeliners and hoses, among others. We received the new experimental results in the area of injection moulding process for the new composites [4] for polymer composites based on engineering plastics/thermoplastics/pigment. Polymer composites was investigated by structural and thermodynamics methods. The planning of the production process must take all these variables into account to obtain a hybrid- and nanocomposites with excellent properties, with low relative cost, and to ensure to fulfil the requirements of the project research and development and approach in industry for the new additive technologies (3-D printing, for example) [3]. We focus our attention on possibilities to develop the innovation hybrid- and nanocomposite materials with predetermined properties based on synthetic polymers with high level elongation at break (at rupture) based our preliminary experience [10] of extrusion of filled in nano-size powder of devulcanized rubber polymer blends (polyolefins / styrene-ethylene-butadiene-styrene, polyolefins / copolymer ethyleneoctene) and hybrid composites based on copolymer ethylene-octene filled in basalt scales. 


\section{Materials and Methods}

Researches are conducted with polymers of thermo elastic type of co-polymer of ultra-low density - copolymer ethylene-octene grade POE SOLUMER 875L obtained by Nexlene technology brand SK Global, density $0,87 \mathrm{~g} / \mathrm{sm}^{3}$; melt flow rate 5,0 g/ $\mathrm{sm}^{3}$, tensile strength $6,1 \mathrm{MPa}$, elongation at break $1000 \%$; basalt scales (sizes $0-80 \mathrm{mkm}, 80-160 \mathrm{mkm}$, 160-300 mkm); nano Nickel; nano Nickel Oxide, nano metals synthesis describe as following.

In the course of the study, $\mathrm{NiSO}_{4}$ and $\mathrm{KOH}$ reagents were used for nano nickel. Precipitation of nickel hydroxide was carried out in a solution containing nickel sulphate and alkali. Production of nickel hydroxide was separated on the Buchner funnel using a vacuum pump. After receiving $\mathrm{Ni}(\mathrm{OH})_{2}$ dried at $60{ }^{\circ} \mathrm{C}$, ground and sifted, washed from soluble salts, and dried again. Nickel nano powder was obtained by reducing nickel hydroxide $\left(\mathrm{Ni}(\mathrm{OH})_{2}\right)$ with subsequent passivation in the atmosphere of technical nitrogen, which contains a small amount of oxygen. The recovery process was carried out at a speed of $10{ }^{\circ} \mathrm{C} / \mathrm{min}$ to $280{ }^{\circ} \mathrm{C}$, followed by exposure for 5 minutes and a decrease in speed by $5{ }^{\circ} \mathrm{C} / \mathrm{min}$ to a temperature of $300{ }^{\circ} \mathrm{C}$ with an exposure of 1 hour. Qualitative X-ray phase analysis of the sample was carried out on a diffractometer "Diffray-401", the survey was carried out according to the Bregg-Brentano scheme. The study of the sample surfaces was carried out using a scanning electron microscope (SEM) "Tescan" "VEGA 3SB". With the help of differential scanning calorimetric and thermogravimetric analyses on the installation of Simultaneous DSC-TGA series Q Series TM SDT Q600, the nature of the recovery of nickel nanopowder was determined. Identification of polymer spent by Fourie-IR-spectroscopy method by VERTEX (Bruker Corporation, USA) in area 350-7500 sm-1 in the oxygen atmosphere of air. Thermostability and heat flow of source samples of the components and obtained composites investigated by methods: thermal analysis (TGA) and differential scanning calorimetry (DSC) and properties of solid samples of Nickel particles, POE SOLUMER 875L and nanocomposites (obtained by melt extrusion) investigated by equipment NETZSCH Erich Netzsch GmbH \& Co. Holding KG, (Germany,) on platinum crucible in area of temperature $0-2400^{\circ} \mathrm{C}$, velocity of the heating of the samples $10^{\circ} \mathrm{C} / \mathrm{min}$. Temperature scale and enthalpy of melting was calibrated by standard sample of In (temperature of melting $T_{\text {melt }}=156,6{ }^{\circ} \mathrm{C}$, specific heat of melting $\Delta H=28,44$ Joule/g). Results were applied for the degree of crystallinity determination. Preparation of hybrids-and nanocomposites was by extrusion and injection moulding method. Temperature regime shown in following Table 1.

\begin{tabular}{|c|c|c|c|c|c|}
\hline \multirow{3}{*}{ Thermo Elastoplast } & \multicolumn{5}{|c|}{ Temperature in cylinder zones of extruder, ${ }^{\circ} \mathrm{C}$} \\
\hline & \multicolumn{5}{|c|}{ Zones of extruder } \\
\hline & 1 zone & 2 zone & 3 zone & 4 zone & 5 zone \\
\hline POE Solumer $875 \mathrm{~L}$ & 160 & 175 & 185 & 190 & 190 \\
\hline
\end{tabular}

Table 1. Temperature zones of extruder for strand obtaining from granules of copolymer ethylene-octene

\section{Results and discussion}

\subsection{The structural characterization of the synthesized nickel nanopowder}

The characterization of the synthesized nickel nano powder (nanoNi), which was later used to produce a nanocomposite, is shown on Figure 1. The study of samples by scanning electron microscopy showed that nanoNi powder is aggregates consisting of densely aggregated spherical particles. Figure 4 shows a micrograph of a nanoNi sample, the average size of which is $29 \mathrm{~nm}$. Based on the obtained microphotographs, a histogram of the particle size distribution is constructed. It can be seen that the histogram of the particle size distribution of the sample obeys the logarithmic-normal law. According to the results of qualitative X-ray phase analysis, it was found that only two phases are present in the nanoNi sample: $\mathrm{Ni}, \mathrm{NiO}$. In this regard, it can be assumed that the nickel nano powder began to oxidize due to high surface activity, which is confirmed by the presence of oxygen of the element according to chemical analysis.

\subsection{POE Solumer $875 \mathrm{~L}$ and filled in composites thermodynamic properties investigation}

Endothermic peak is pronounced on the DSC the curve of the sample POE SOLUMER 875L on Figure 2 in area of temperatures $30-80{ }^{\circ} \mathrm{C}$. with significance of the value of the enthalpy of formation $-41,33 \mathrm{~J} / \mathrm{g}$ [8] responds to the melting process (it is known that the temperature of melting of investigating copolymer POE SOLUMER $875 \mathrm{~L}$ respond $61{ }^{\circ} \mathrm{C}$.

Strand of nanocomposite (POE SOLUMER 875 L/0,5 \% mass. nano Ni and strands of hybrid composites (POE SOLUMER 875 L/Basalt scales) obtained by mould extrusion method. Further DSC the curve obtained for several samples of composites based on nanocomposite $0.5 \%$ mass..nano Ni /POE Solumer 875L and composites filled in basalt scales of different dispersion (0-80-160-300 mkm) and content 2,5\% mass.; 5,0\% mass.and $10 \%$ mass. in POE SOLUMER 875L. Some of results of DSC analysis - shown further (on Figures 8 and 9) - demonstrate that extremum of melting process shifted and smoothed to area more high temperatures (about $90-100{ }^{\circ} \mathrm{C}$.), that testifies of shifting of melting process to higher temperature the new obtained nanocomposite containing nano nickel and composites based on POE SOLUMER $875 \mathrm{~L}$ filled in basalt scales 


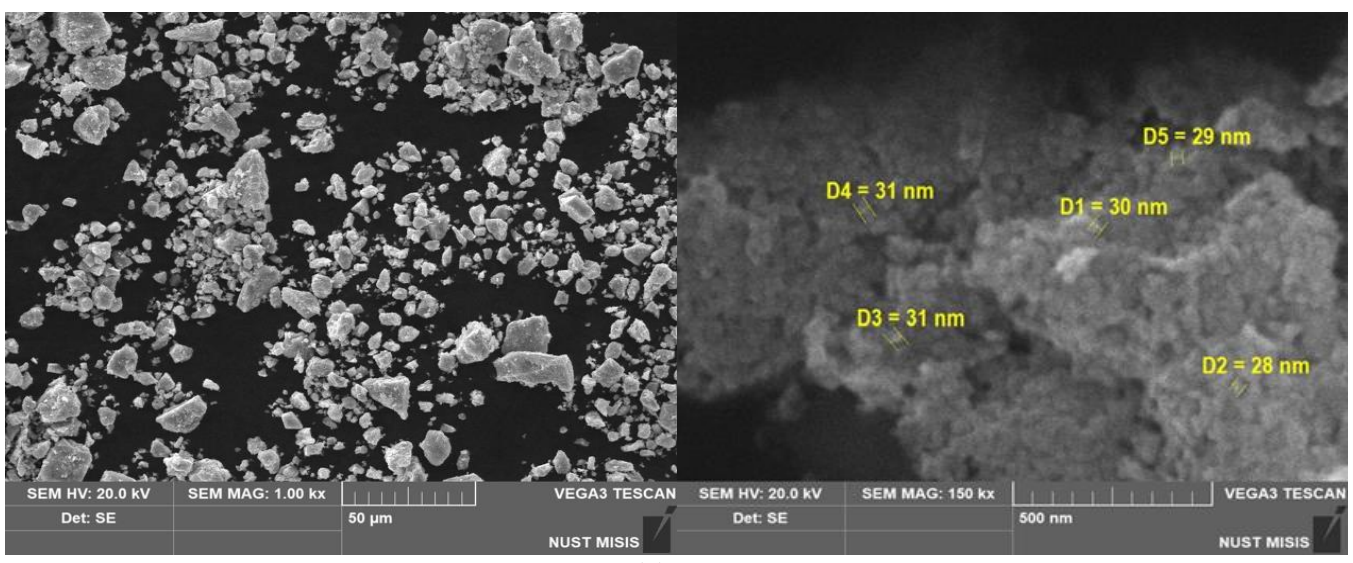

(a) (b)
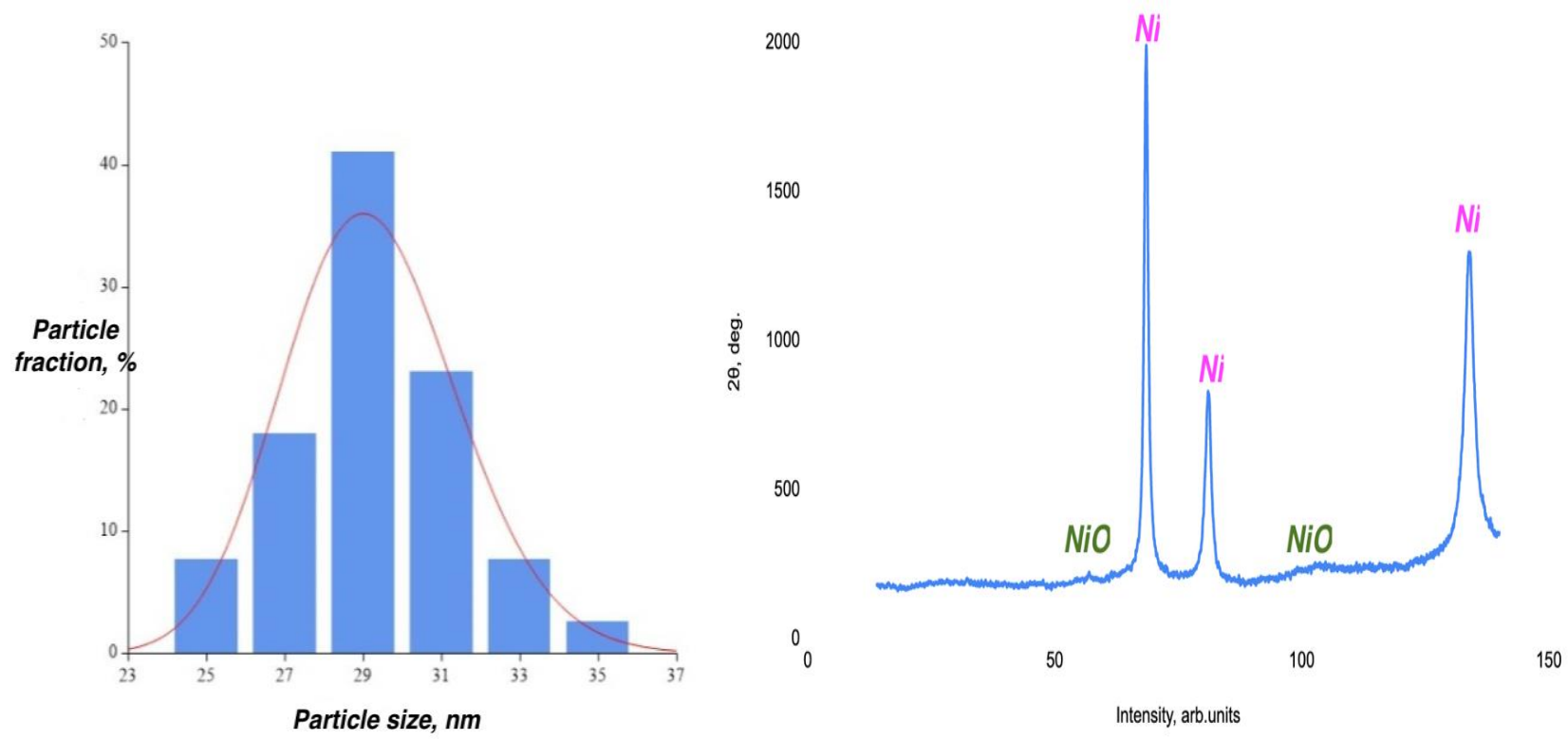

(c)(d)

Fig. 1. Characteristics of nickel nanopowder:.(a,b) Microphoto of nanoNi;(c) Histogram of the particle size distribution of the nanoNi, (d) XRD patterns of NanoNi

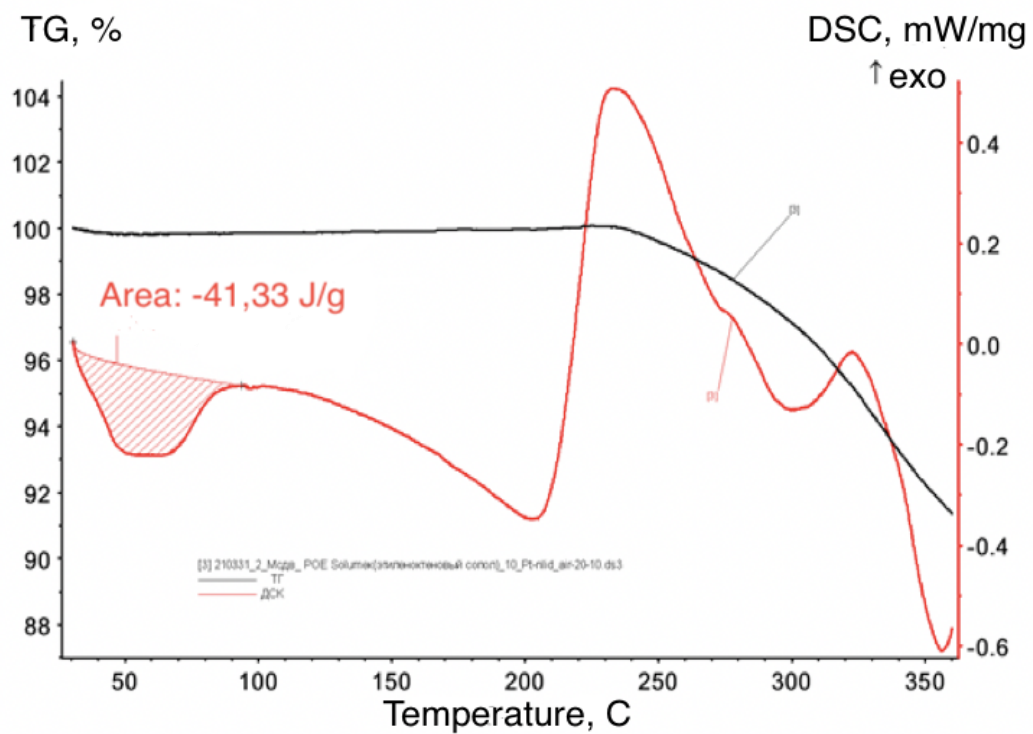

Fig. 2.TGA curve of the ethylene-octene copolymer in the temperature range $20-350{ }^{\circ} \mathrm{C}$ (black, 2), DSC curve of the ethylene-octene copolymer in the temperature range $20-350{ }^{\circ} \mathrm{C}(\mathrm{red}, 1)$ 


\subsection{Nanocomposites based on POE Solumer/0.5\% mass.. nanoNi and hybrid-composites POE Solumer/Basalt scales:structure, thermostability and elasticity}

Strands obtained by melt extrusion of filled in nano nickel and basalt scales of sizes mentioned above in part 2 Materials and methods [11] shown below on photo (Figure 3 and Figure 4). We investigated structural, thermodynamics, and physico-mechanical properties of the samples of obtained composites in film form and strands by several experimental methods described above in Part 1.

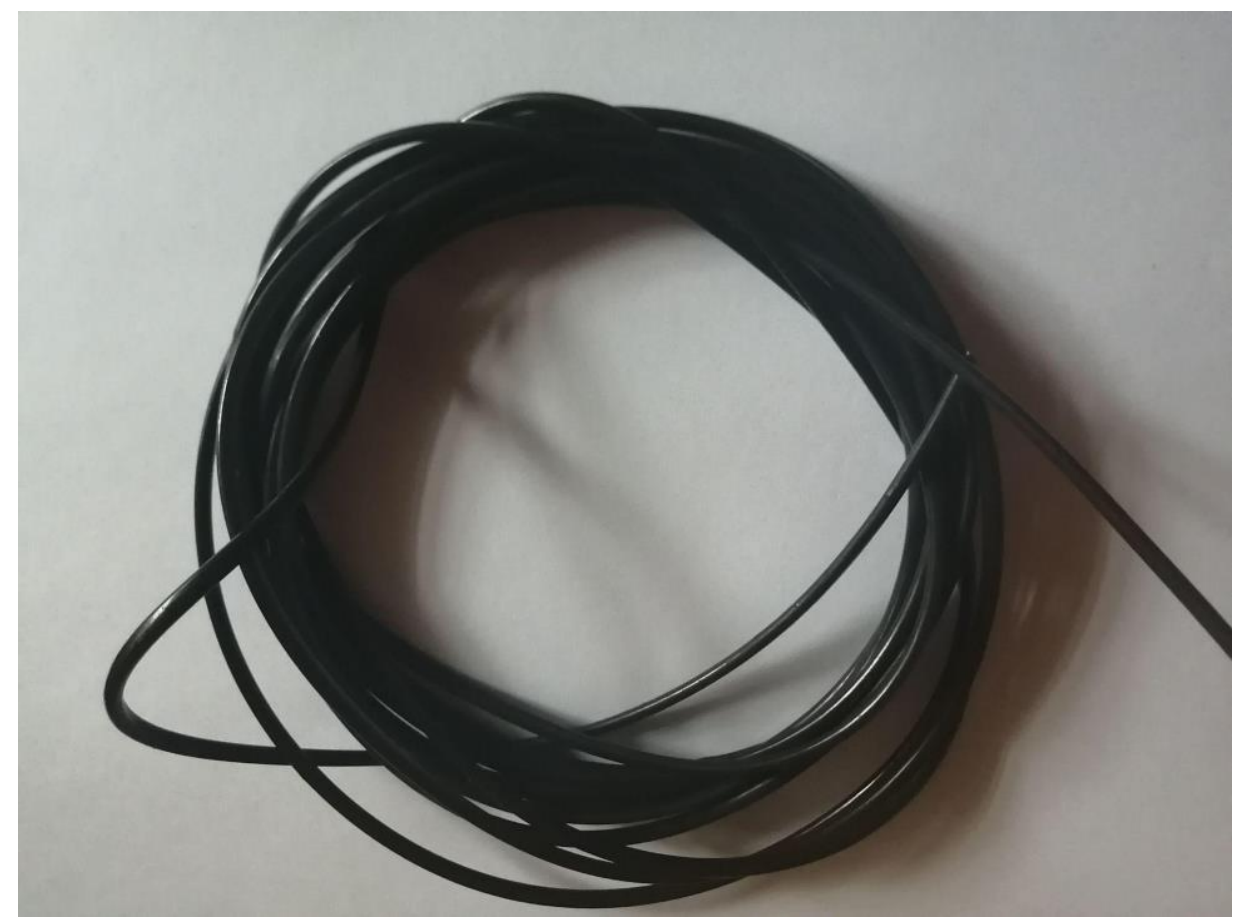

Fig. 3. Foto of strand nanocomposite (POE SOLUMER 875 L/0,5 \% mass nano Ni) obtained by melt extrusion method

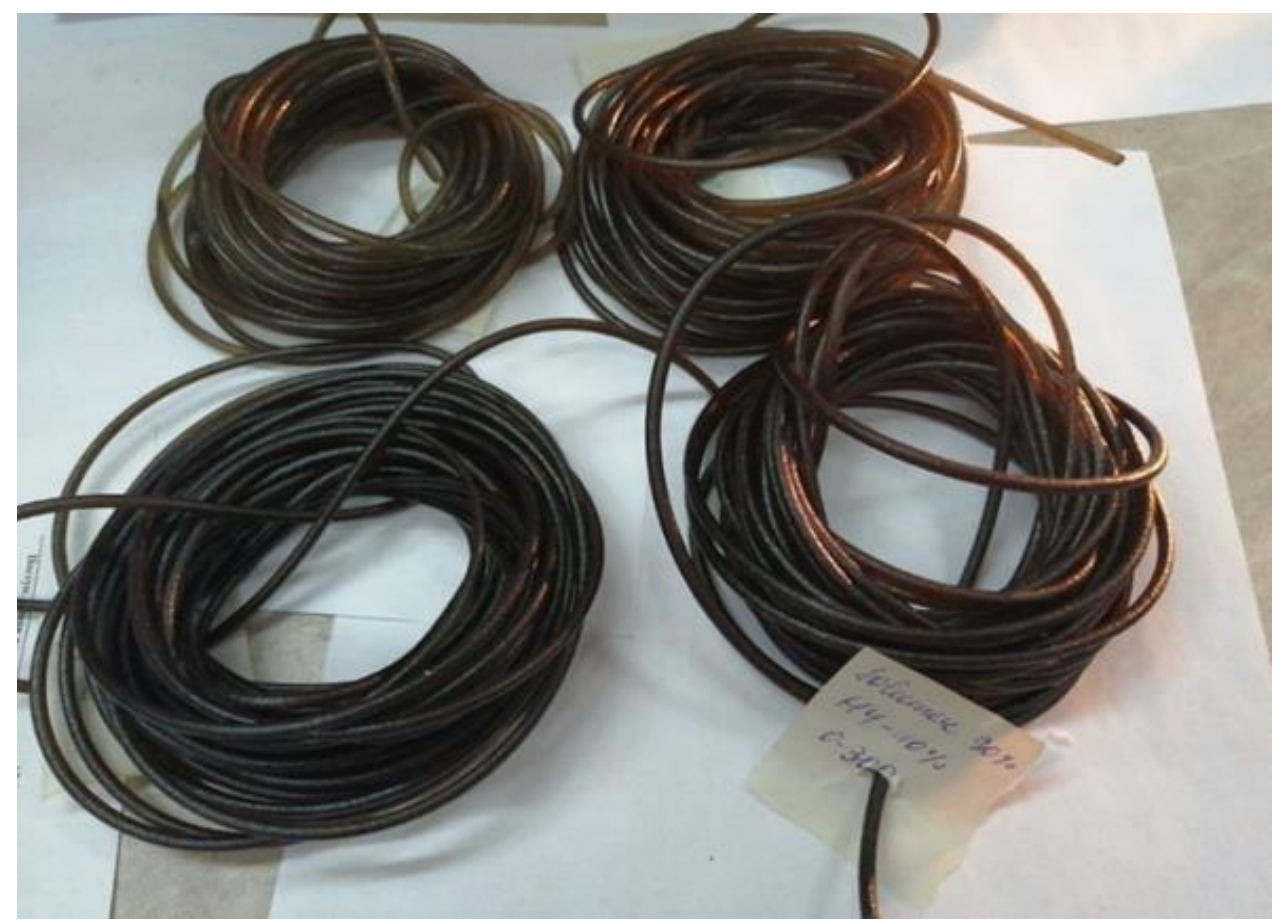

Fig. 4. Foto of strands hybrid composite (POE SOLUMER 875 L/Basalt scales) obtained by extrusion method

Qualitative X-ray diffraction analysis (Figure 5) showed that two phases are present in nanocomposite samples: nickel phase and $\mathrm{C}-\mathrm{H}$, which corresponds to this copolymer, POE SOLUMER 875L. 


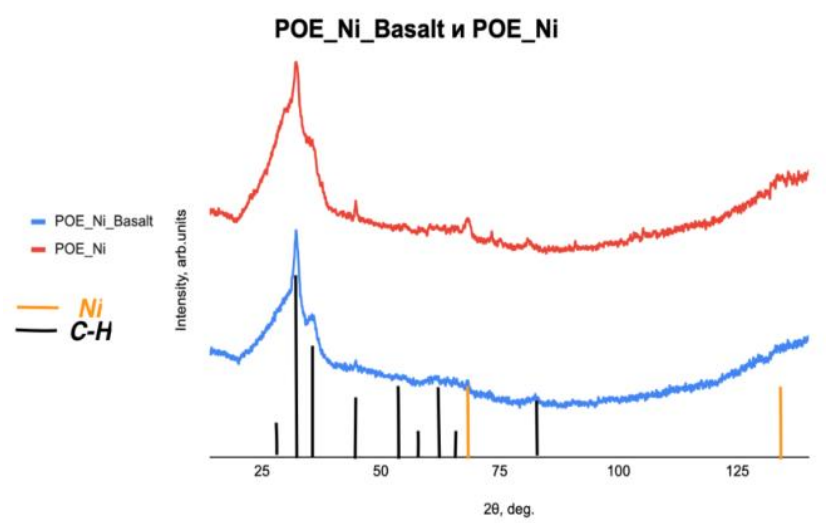

Fig. 5. XRD patterns of POE Solumer 875L filled in blend of nano Ni and Basalt Scales and POE Solumer filled in nano Ni powde

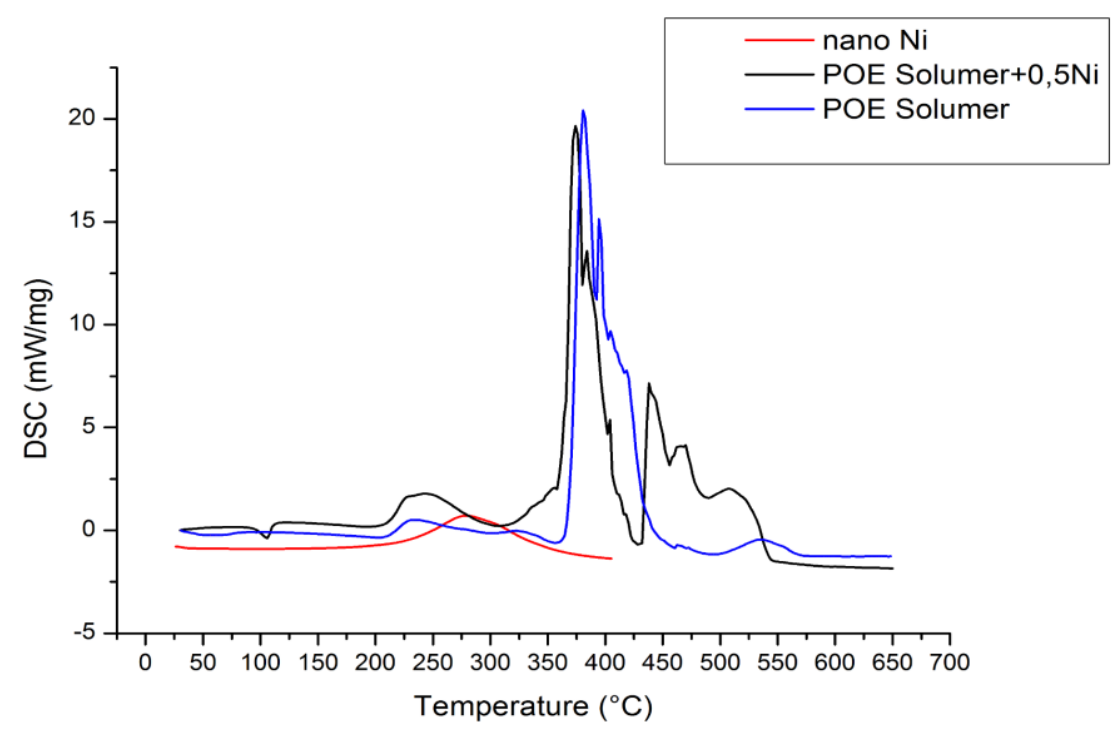

Fig.6 DSC analysis of samples of a nano Ni, POE Solumer 875L, nanocomposite $0.5 \%$ wt. nano Ni /POE Solumer $875 \mathrm{~L}$

DSC analysis of the samples of samples of a nano Ni, POE Solumer $875 \mathrm{~L}$, nanocomposite $0.5 \%$ wt. nano Ni /POE Solumer 875L represented on Figure 6. Figure 7 and 8, 9 show the results of comparative investigation of the loss of mass of the samples thermo elastoplast POE SOLUMER 875, nanocomposite $0.5 \%$ wt. nano Ni /POE Solumer $875 \mathrm{~L}$ and hybrid composites with different content of basalt scales. It is found that the thermostability of nanocomposite is higher than thermostability of polymer.

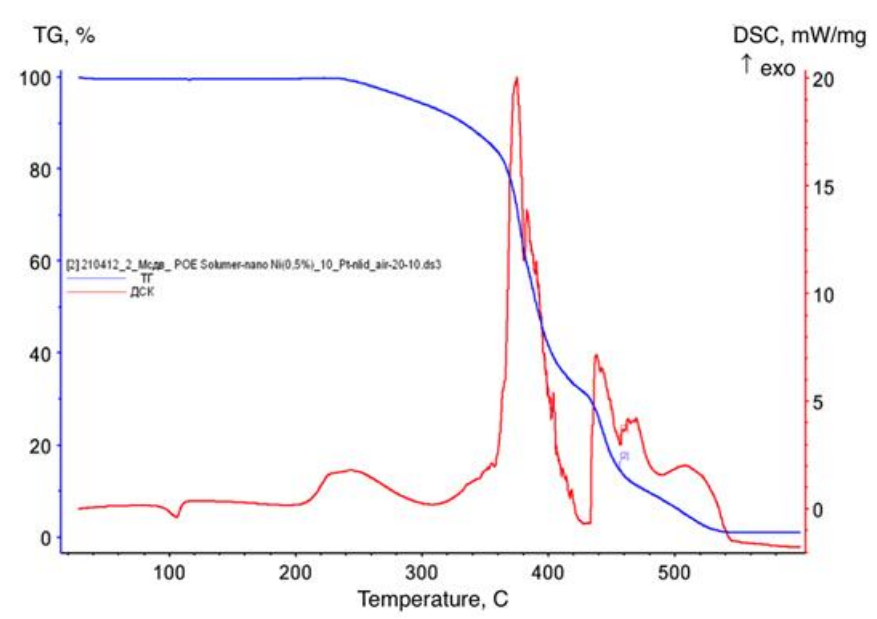

Fig. 7. TGA/DSC analysis of the sample of nanocomposite $0.5 \%$ wt. nano Ni /POE Solumer $875 \mathrm{~L}$ 


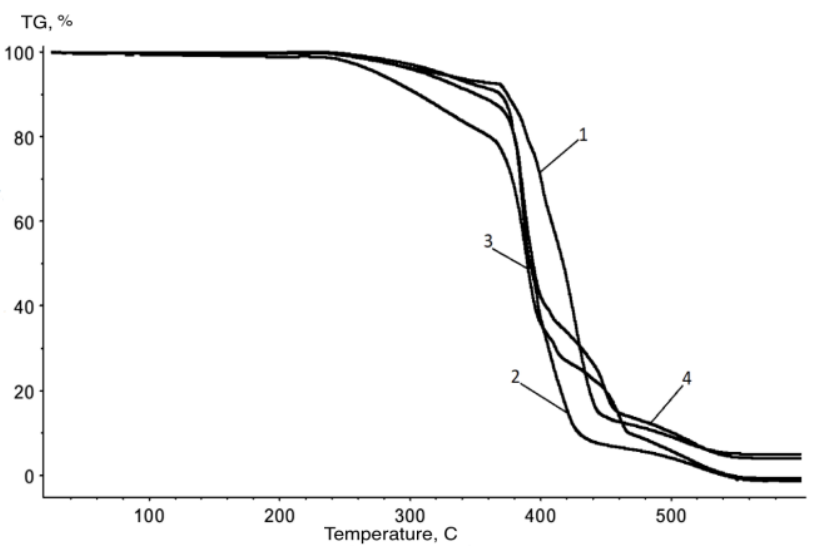

Fig. 8. TGA curves of a sample POE SOLUMER 875L (2) and hybrid composites filled in basalt scales sizes 80-160 mkm: $10 \%$ mass.(1), $2,5 \%$ mass.(3), $5 \%$ mass.(4)

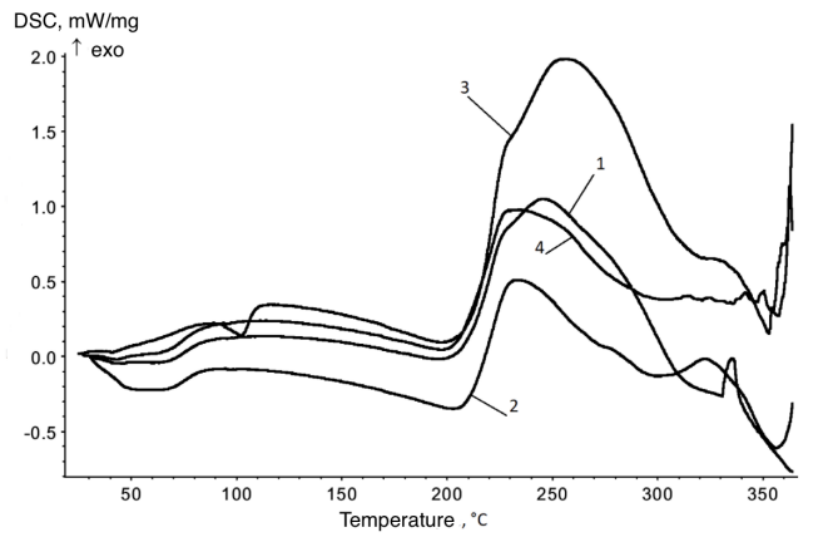

Fig. 9. DSC curves of a sample POE SOLUMER 875L (2) and hybrid composites filled in basalt scales sizes 80-160 mkm: $10 \%$ mass.(1), $2,5 \%$ mass..(3), $5 \%$ mass $/(4)$

The most important result of physico-mechanical properties investigation (by methods described above, in part 1 of this paper) is increasing tensile strength of elasticity and of the new composites: tensile strength value (when breaking) of the sample POE SOLUMER 875, as known, equal to 6,1 MPa) and the tensile strength hybrid-composites samples of copolymer containing basalt scales growing to7 MPa: tensile strength of the samples of nanocomposite nano Ni/POE Solumer 875L became $10 \mathrm{MPa}$; elongation of break of the samples of nanocomposites (from 1000\% for POE SOLUMER 875L to $1240 \%$ for nanocomposite sample nano Ni//POE Solumer $875 \mathrm{~L}$ ).

\section{Conclusion}

Propositions of the paper as well as the author's views of the practical implications of the results for increasing elasticity of the new hybrid- and nanocomposites based on polymers type thermo elastoplasts filled in basalt scales and nano powder of metals. The most important results of presented investigations is obtained by reinforcement of investigated thermal elastoplast, (ethylene-octene copolymer) by coextrusion with nanoparticles powder ( $30 \mathrm{~nm}$ ) of metals and its oxides. Determination of improve the open possibilities to obtain the new hybrid- and nanocomposite materials based on ethylene-octene copolymer filled in basalt scales and nanomaterials and metal oxides with predetermined properties for industrial approach for the new goods manufacture in form films, fibres, strands, hosepipes and the other forms with higher elasticity than initial polymer. Conducted research and new results open the possibility of application of the new hybrid- and nanocomposites based on thermoplastics filled in basalt scales, nano metals powder and their blends for application for increase of tensile strength and elongation of break. in additive technologies such as 3D-printing.

\section{Acknowledgments}

Research and development of presented paper supported by Programme 35 of the Presidium Russian Academy of Sciences. A Scientific Basis to develop New Functional Materials state order No 0082-20019-0008 Developing "Methods to make Nanostructured Polymer, Biopolymer and Composite Materials and their Target Modification Aimed at Wide Range of Practical Applications". 
An experimental investigation was conducted with the experimental support of y Grachev A.(Senior researcher FRC ChPh NN Semenov RAS) and Golobokov D. (student of Polytech University, Moscow) CCP №5006694 and UNU № 144073 of the Federal Research Centre of Chemical Physics Semenov NN of the Russian Academy of Sciences.

\section{References}

[1] Hodzik et al.(2020) Strain Rate Influence on Mechanical Characteristics of FDM 3D Printed. Materials. proceedings of 32th DAAAM International Symposium, B.Katalinich (Ed.), Vienna, Austria, ISBN 978-3902734 http://dx.doi.org/10.2507/31st.daaam.proceedings.02

[2] Myasoedova V.V. and Taran I.A. (2020) Chemistry of Energy-Effective Polymer Nanocomposites (ID Academia Zhukovskogo, Moscow, 212 p.(in Russian), ISBN 978-5-907275-12

[3] Fedorov L.Yu.(2017) DSC/TGA Analysis of composite material based on highest density polyethylene and nanoparticles CuO, POLYCOM TRIB-2017, Gomel, Belorussia, June, 27-30, pp.-20-23

[4] Svoboda P.,TherraValappil R., Svobodova D. et al. (2010) Elastic properties of polypropylene/ethylene-octene copolymer blends.Polymer Testing, Vol.129, Issue 6, September, pp.742-748

[5] Myasoedova V.V., Taran I.A., Lushkova A.V. Physical and Chemical Properties of Composites Produced on Disperse-filled Blends of Polymers , Polymer Science, Series D, 2019, Vol.12, No 2, pp.203-206, ISSN 1995-4212, DOI:10.1134/S1995421219020151

[6] Keihan A.N. Veisi H., Veasi H (2017) Green synthesis and characterization of spherical copper nanoparticles as organometallic antibacterial agent, Appl.Organomet.Chem, https://doi.org/10.1002/aoc.3642

[6] Dahoumane S.A. et al (2017). Biosynthesis of inorganic nanoparticles: A fresh look at the control of shape, size and composition, Bioengineering. https://doi.org/10.3390/bioengineering 4010014

[7] Drexler R.T. (1981) Molecular engineering: an approach to the development of general capabilities for molecular manipulation. Proc Natl 1Ab afSci. USA78, p.5275

[8] Myasoedova V.V. (2018) Recycling of different chemical nature polymers for approach as composites (Plenary lecture) //The Book of the Fourth International Conference on Reuse and Recycling of Materials, ICRM-2018, March 09-11, pp.12-15

[9] Svoboda P.,TherraValappil R., Svobodova D. et al. (2010) Elastic properties of polypropylene/ethylene-octene copolymer blends.Polymer Testing, Vol.129, Issue 6, September, pp.742-748

[10] Myasoedova V.V. Lushkova A.V.(2020) Eco solution: composites based on recycled polypropylene modified by thermoelasoplasts and filled in disperse filler. CHEMICAL SAFETY SCIENCE, No 4(2), pp.265-281

[11] Myasoedova V.V.(2021) Graded Recycled Polymer Modified by Thermoelastoplasts: Composite Materials to be Used for Injection Molding, Pol. Sci., Series D, Vol.14, No 3, pp. 462-466, ISSN 1995-4212, DOI:10.1134/s1995421221030217 